\title{
NUMERICAL AND EXPERIMENTAL STUDY ON TSUNAMI RUN-UP AND INUNDATION INFLUENCED BY MACRO ROUGHNESS ELEMENTS
}

\begin{abstract}
Nils Goseberg ${ }^{1}$ and Torsten Schlurmann ${ }^{2}$
This research study considers long wave run-up experimentally and numerically. At first, an alternative methodology in long wave physical modeling is presented by means of a set of pipe pumps forcing the inflow of a controlled volume of water into a wave channel mimicking a tsunami-like wave shape that is consistently contained by a proportional plus integral plus derivative controller (PID) controller. Arbitrary wave lengths are persistently generated by means of the proposed methodology. First results are compared to tsunami data stemming from conventional experimental configurations with solitary waves as well as with recent numerical modeling results. Comparisons are thoroughly discussed and - in a second step - numerical simulations are accomplished taking the interaction of long wave run-up and macro-roughness elements into account. Four different experimental configurations of macroroughness elements are carried out while spacing between elements and numbers of obstacle rows are alternated. A fundamental correlation analysis reveals that a correlation of the number of macro-roughness rows, effective area of flow cross section and a grouping factor of different element configurations exists in principle.
\end{abstract}

Keywords: long wave; tsunami run-up; macro-roughness; numerical modeling; physical modeling; laboratory wave generation

\section{INTRODUCTION}

In the context of many disastrous tsunami incidents such as the Indian Ocean tsunami in 2004 or the Aleutian tsunami in 1946, the understanding of tsunami deep water propagation and transformation in shallow waters has grown rapidly in the last century. Nevertheless, the understanding of tsunami run-up dynamics and coastal inundation is still limited. Especially in coastal urban agglomerations, where the tsunami induced on-land bore-like wave front patterns are strongly dominated by macro roughness elements such as houses and infrastructure. This inundation process is difficult to model both physically and numerically (Cox et al. 2009).

This paper focuses on the interaction between tsunami run-up and macro roughness elements in a very conceptual approach applying numerical modeling techniques along with basic physical model tests. The influence and interdependencies of a varying obstruction factors and macro roughness elements are discussed for a realistic tsunami-shaped wave form to be applied in forthcoming numerical investigations.

\section{BACKGROUND AND OBJECTIVE}

When a tsunami approaches coastal waters, wave deformation takes place due to varying water depth. The shoaling wave is characterized by amplification of the wave height while wave length is reduced. Potentially, the approaching wave may break before inundation takes place onshore. Based on the coastal topography on-land flow is also possible. Generally, maximum inundation distance in the coastal hinterland depends in first order on the slope of the near coast region as well as on land cover characteristics. Wave reflection is augmented proportionally to the bottom slope and similarly, dense coastal vegetation, i.e. marshes or mangroves, and infrastructure and municipal developments influence both inundation dynamics and run-up distances.

Those phenomena in the near-shore region related to long wave run-up are often captured by numerical or physical models since in-situ field observation of real tsunami run-up mostly contain sparse but also retrospective observation points. In regard to physical tsunami models only few studies link up length scales in model tests and prototypes (see e.g. Briggs et al. 1995). Solitary waves are often associated with tsunami evolution in deep water and during propagation. Likewise, they are also deployed in physical model test to investigate run-up and run-down behavior (Synolakis 1986). Once near shore environments are experimentally modeled, the key question arises if solitary waves are a valid choice to mimic tsunami in order to reflect the prototype wave characteristics correctly (Madsen, Fuhrman and Schäffer 2008).

The application of conventional numerical tools for large scale inundation studies, which usually solve nonlinear shallow water wave equations (NSWE), is accompanied by two major challenges. In first instance, bottom roughness mostly formulated according to Manning's n value or Chezy coefficient yield first order sensitivity when the wave induced current propagates on-land over a flat coastal

\footnotetext{
${ }^{1}$ Research Associate and PhD candidate, Franzius-Institute for Hydraulic, Waterways and Coastal Engineering, Leibniz Universitaet Hannover, Nienburger Str. 4, Hannover, 30167, Germany, Email: goseberg@fi.uni-hannover.de

${ }_{2}$ Managing Director and Professor, Franzius-Institute for Hydraulic, Waterways and Coastal Engineering, Leibniz Universitaet Hannover, Nienburger Str. 4, Hannover, 30167, Germany, Email: schlurmann@fi.uni-hannover.de
} 
area. Furthermore, so-called macro-roughness elements (Cox et al. 2009) such as buildings and infrastructure interact with the transient on-land flow induced by the long wave. This interaction causes significantly higher water surfaces elevation in front of the constructed environment whereas maximal inundation distances are reduced remarkably (Taubenböck et al. 2009).

The objective of this study is therefore to firstly propose an alternative long wave generation methodology. Secondly, the effect of macro-roughness elements to the long wave run-up should be examined more closely by means of physical as well as numerical modeling. Substantially longer (realistic) waves are considered which are in the order of magnitudes of measured wave time histories (e.g Mercator Yacht echo sounder, see Ward and Day 2008). Comparisons between solitary waves and realistic near shore waves are accomplished subsequently. Furthermore, wave run-up and wavestructure interaction is computed by utilizing a NSWE model (Nielsen et al. 2005). Both approaches are intended to gain insight into the various effects influencing wave run-up dynamics in densely populated coastal areas where on the one hand tsunami induced flow fields in the constructed environment are retarded and on the other hand flow velocities are amplified due to Venturi-like effects in the urban street network (Goseberg et al. 2009). Finally a correlation between macro-roughness configuration and wave run-up is proposed, which relates dimensionless obstruction factors to the long wave run-up.

\section{PHYSICAL MODELLING}

Appropriate long wave generation is crucial when it comes to experimental studies of tsunami related to wave run-up and inundation dynamics in the context of urbanized coastal settlements. The generation of commonly applied solitary waves is straightforward (Galvin 1964) and widely applied in coastal labs worldwide, but especially near-shore tsunami waves are reported to exhibit much longer periods than periods originating from solitary waves, e.g. referring to typical laboratory length scales of 1:100. Therefore a new wave generation is proposed in this study.

\section{Wave generation methodology}

Long wave generation is achieved by means of two high-capacity and synchronously driven pipe pumps, which are fitted into a closed-circuit flume. The flume is schematically illustrated in figure 1 . It has been originally constructed and applied to investigate long-term sediment suspension studies in unsteady flow fields. Therefore controlled amounts of water volumes in the $65.00 \mathrm{~m}$ long flume can be arbitrarily accelerated or decelerated at the pump station (a).

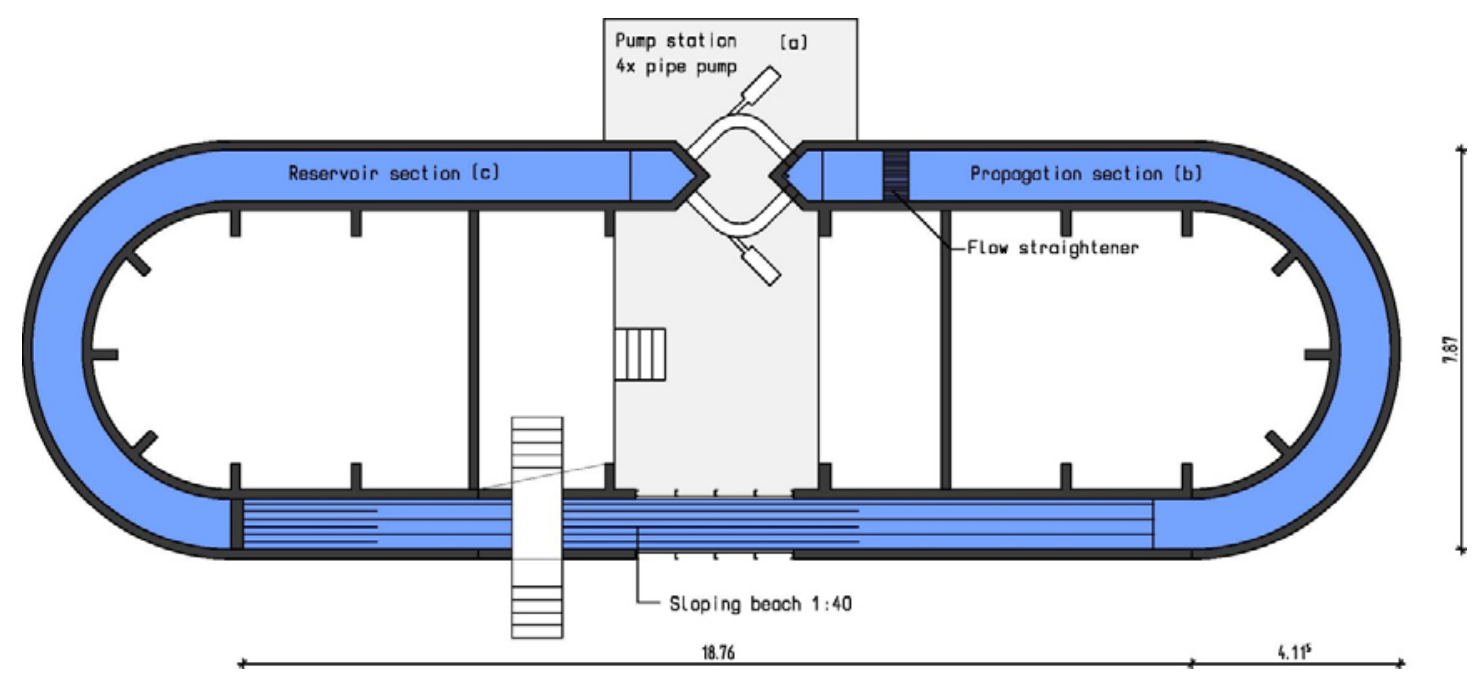

Figure 1. Schematic drawing of the closed-circuit flume: (a) Pump station with four high capacity pipe pumps, (b) propagation section, $180^{\circ}$ degree bend and the sloping beach, (c) reservoir section storing water volumes

In contrast to the sediment suspension experiments where an infinite closed-circuit flume is demanded, the flume was divided into two sections, i.e. a wave propagation section and reservoir section to store sufficient water volumes in order to generate a tsunami-like wave front in the flume. A rigid lime sand brick wall of $0.55 \mathrm{~cm}$ height separates effectively both sections. At the straight flume section at the bottom of figure 1, a 1:20 and 1:40 flat sloping beach has been installed, which is covered by floating pavement and at a later stage by PVC board. 
A set of practical straightening measures at the out- / inlet of the pipes into the flume attenuates undesirable oscillations and turbulences effectively. Various sensors such as pressure sensors, capacity wave gauges, ultrasonic distance sensors, electro-magnetic velocimeter and CCD camera are installed along the beach profile to measure a full set of hydrostatic and hydrodynamic quantities.

The pipe pumps are driven by means of a proportional plus integral plus derivative controller (PID controller). Scaled tsunami sea surface height (SSH) and sinusoidal wave time series are used as target value time signals. Wave periods in the flume are in the range of $\sim 60.00 \mathrm{~s}$ to $100.00 \mathrm{~s}$. The PID controller processes the instantaneous value input of the water level measured in the vicinity of the pump outlets into the flume (see figure 1, position (d)). The methodology of controlling the experimental performance of the long wave generation routine is depicted in figure 2. The PID controller generating the wave signal is additionally capable of absorbing reflected waves stemming from the wave rundown on the sloping beach, since instantaneous deviations from the initial target value of the artificially generated long wave signal implicitly leads to appropriate in-/outlet adjustment of the pumps.

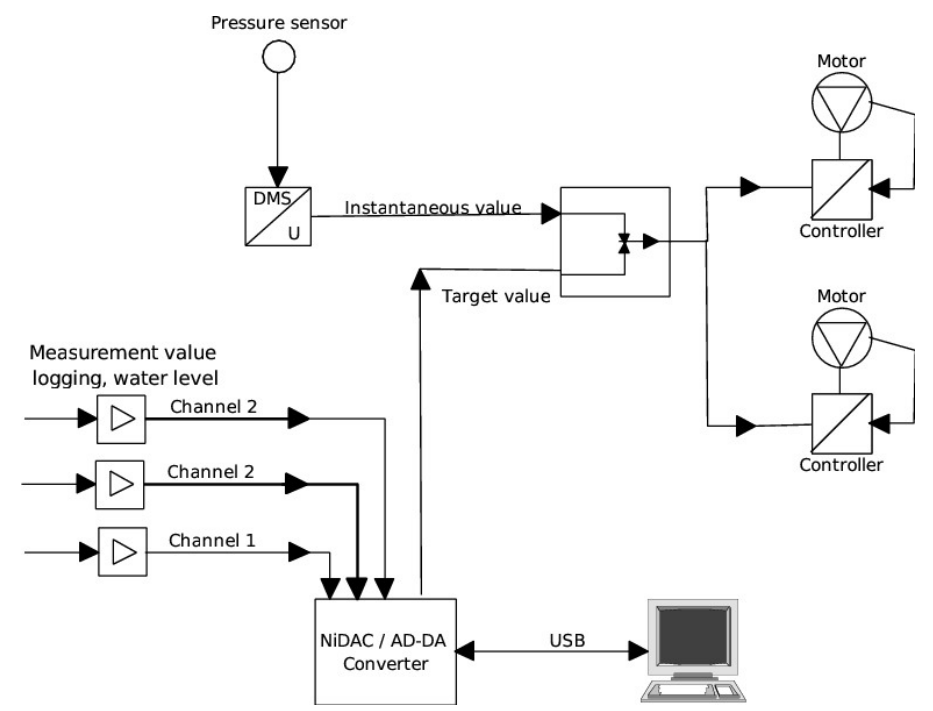

Figure 2. System sketch of connections for the applied controller set-up with PID controller, wave gauges, pressure sensor and flow of data

PID-controllers belong to the group of feedback systems, which rely on a continuous compensation between a given target time series and an instantaneous value. Depending on the gain of each of the individual proportional, integral and derivative parameter, oscillations may increase according to the gain level. In order to reduce the error or reset, controller parameters need to be adjusted until an optimum is reached or at least satisfactory results are obtained. Appropriate PID tuning may be simplified by obeying tuning rules, e.g. the Ziegler-Nichols method (Åström and Murray 2008). Here, manual adjustment of the controller in conjunction with an optimization of the inlet geometry yields minimum error between target time series and instantaneous sensor values at the inlet for the experimental setup.

\section{Capabilities and limits}

Figure 3 shows the target signal history (dash-dotted line) with the resulting wave being generated. It depicts the comparison of a complex target signal taken from hydro numerical simulation at Padang, West Sumatra (Goseberg and Schlurmann 2009) and the resulting time series from the laboratory measured at the outlets of the pumps. A signal with higher frequency is modulated on top of the basic signal resulting from the PID feedback control in combination with disturbances of the water surface at the pressure sensor location due to the controller scheme. The feedback control also yields an evident time shift between target signal and generated signal. This resembles the apparent retard of the implicit long wave generation routine. Nevertheless general agreement between both time series is remarkably good. High frequency bands in the wave history have only little effect on the maximum wave run-up whereas lower frequencies contribute to first order of magnitude to the net shoreward mass transport responsible for the often massive observable wave run-up in nature. Even though the generated time series shows minimal distortions, the proposed methodology seems to be a valuable tool in generating and investigating long wave run-up in laboratory environments. The RMS error for the generated time 
series comprises $0.67 \%$ related to the target value time series over the depicted dimensionless time $\mathrm{t}^{*}(\mathrm{~g} / \mathrm{s})^{\wedge} 0.5=13$.

Regarding the given closed-circuit flume geometry, wave propagation along the flume and especially through the curvature of this experimental facility results in augmented loss of energy. Moreover, while the long wave propagates along the curvature of the flume continuous reflection takes place leading to unwanted secondary flows superimposed on the primary flow. This hydraulic artifact is beyond any doubt a legitimate experimental limitation of the current closed-circuit flume geometry and is being dealt with correctly in present experimental investigations carried out these days.

An upper limit for persistently generated wave periods is obviously given by the maximum storage volume of the reservoir section. Furthermore, the long wave surface elevation gradient is limited by the total hydraulically realizable discharge as a sum of both pipe pumps reduced by the energy dissipation at the inlet.

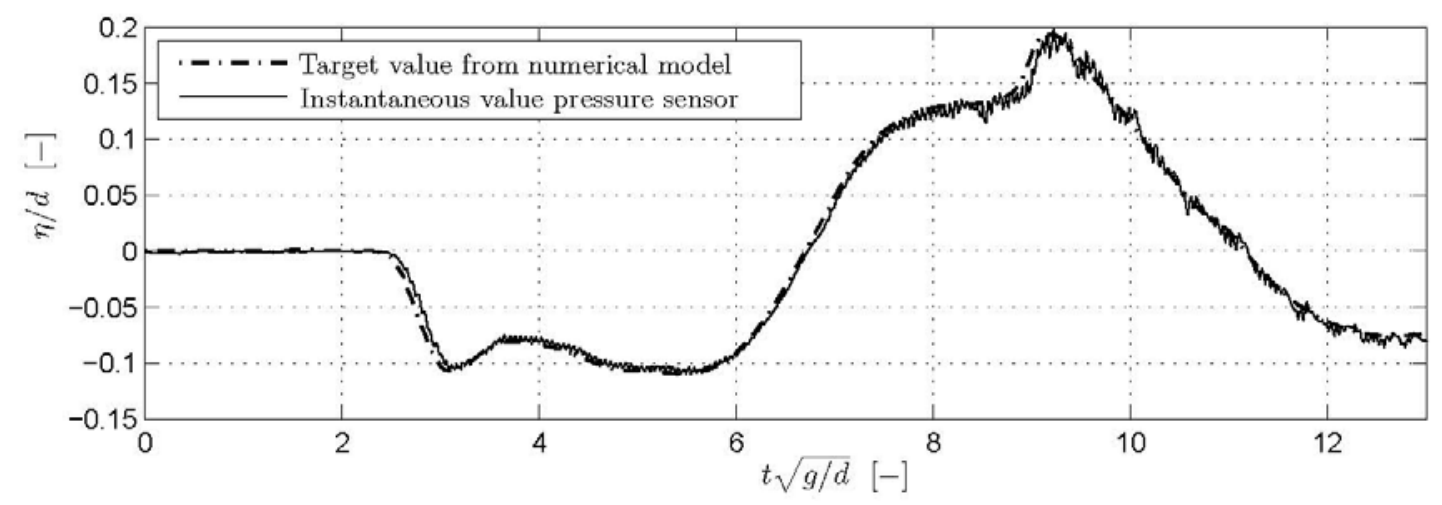

Figure 3. Resulting time series target value (dash-dotted line), instantaneous value at the pump outlet (solid line) in dimensionless form for tsunami wave shape from numerical modeling, $n$ free surface, $d$ local water depth, $g$ earth acceleration

Finally, figure 4 illustrates two long wave time series - a first order solitary wave and a wave generated in the laboratory by means of the proposed methodology. The graph reveals that the wave generated by the new experimental methodology displaces a significantly larger volume of water than the ordinary solitary wave generation approach. Thus, better comparability of laboratory experiments to the modeled prototype situation is expected from the presented methodology.

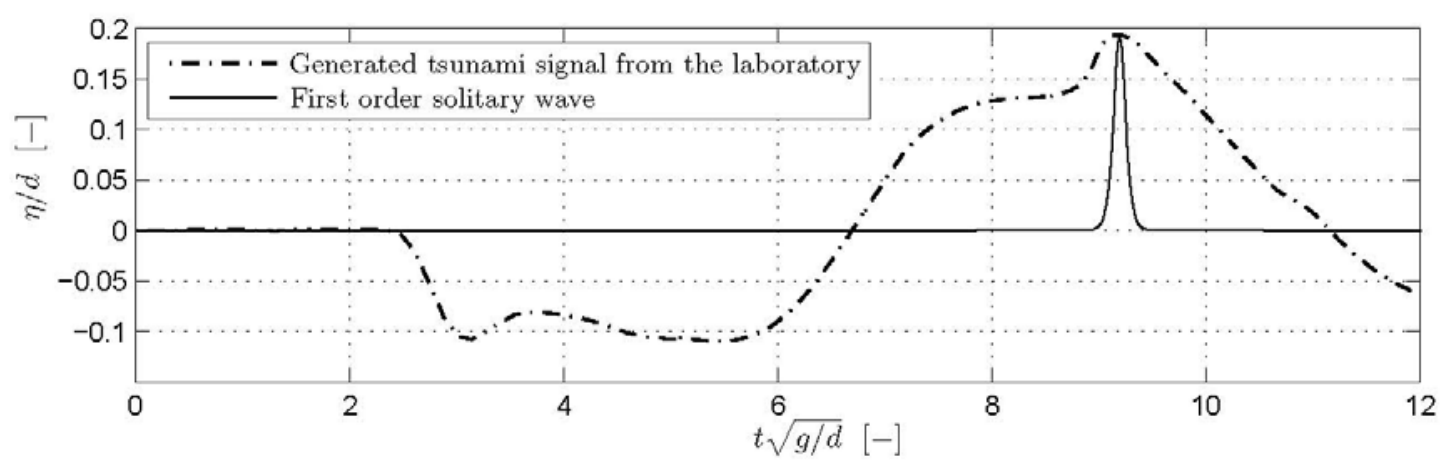

Figure 4. Time domain comparison of a first order solitary wave and the experimentally generated wave used for the current study with identical wave height and water depth 


\section{NUMERICAL MODELLING}

\section{Modeling macro-roughness elements}

The implementation of vertically orientated structures such as buildings, natural and artificial barriers, dikes or embankments into numerical models consisting of a depth averaged equation solution becomes challenging since discontinuities in the bottom elevation are generally not captured well. Nevertheless, three dimensional models are less feasible in terms of computational cost when it comes to large scale modeling aiming at hazard assessment and risk mitigation. However, two and three dimensional strategies to cope with macro-roughness elements are documented in literature, which are mentioned here for sake of completeness and for better orientation of the presently proposed approach.

At first, Pedro et al. (2007) calculated wave induced forces to a square cylinder with two different turbulence models and validated these results against a laboratory experiment making use of a dam break generated bore. While general surface elevation and fluid forces to the obstacle resemble the experimental data sufficiently, flow velocities are underestimated even by computationally expensive large eddy simulations. Tomita, Honda, and Kakinuma (2007) reported on a study that combined a quasi three dimensional model for ocean wide wave propagation with a fully 3D model in the vicinity of the city of Galle, Sri Lanka in order to investigate the impact of the disastrous Boxing Day tsunami in December 2004. They found good agreement with field measurements. Computational workloads were not discussed in the paper. Likewise, Cox et al. (2009) present a comparative investigation including a 1:50 scaled physical and, subsequent, numerical model yielding excellent agreement between both approaches. An error function for maximum stroke as well as a solitary wave has been applied during the experiments. The employed numerical models were COULWAVE (Lynett, Wu, and Liu 2002) and STOC (Tomita, Honda and Kakinuma 2007). Xiao and Huang (2008) modeled wave run-up and forces to a building wall by means of a 3D numerical VOF formulation and carefully validated the model results with experimental solitary wave run-up data (Synolakis 1986). The computational domain consisted of a cross-sectional slice perpendicular to the shore line. Therefore, spatial effects around the idealized beachfront houses and other infrastructure elements are neglected. However, this study also depicts an important step in addressing fluid-structure interaction of long wave run-up in the near- and onshore region. Various authors also applied depth-integrated equations such as Boussinesq or nonlinear shallow water wave equations incorporating the macro-roughness elements influence on the general flow pattern into account. More recently, the effect of coastal dunes to the run-up and rundown process was highlighted at the Sri Lankan coast (Witejunge 2010). Higher peak flow rates and flow depth arise in the numerical model that is meant to be due to the presence of sand dunes. However, sand dunes impose larger length scales in addition with smoother surface gradients into consideration compared to houses and building which generally miss out the strict verticalness of walls or other flow obstacles. Hashimoto and Park (2008) introduced a set of depth-integrated equations that in addition contain an empirical parameter expressing the area density of houses. The set of equations was applied to a flooding event at Fukuoka city, Japan. The discretized resistance terms include drag forces on the houses and buildings in addition to the usual bottom shear stresses. By deliberately fitting the adequate empirical parameters, good agreement with measured data was achieved in context of urban flooding.

Furthermore, a dam-break event induced flood wave surging through an idealized city was studied experimentally and numerically by Soares-Frazao and Zech (2008). The experimental results were subsequently compared to a depth-averaged model that captured the idealized houses by an impervious boundary condition in order to properly account for reflection behavior at the house walls in the model. Generally, good agreement was found even for only few mesh cells replicating the existing road space. Additionally, macro-roughness and the inherent dissipation of kinetic energy could be modeled by adding artificial porosity in the transient flow regime (Soares-Frazao et al. 2008). Macro-roughness elements could also be modeled by utilizing highly-resolved digital surface models (Goseberg and Schlurmann 2008, 2009). Carefulness is demanded to assure geometrical correctness when areas which are abundantly covered with vegetation are situated inside the model domain. The direct implementation of macro-roughness elements in the model domain is advantageous in cases where the underlying geodata set is finer resolved than individual roughness elements and when detailed information on the temporal distribution for evacuation planning is demanded. Apart from using surface models, one may utilize digital elevation models without houses and building on a larger scale of modeling. In this case, energy dissipation in the highly non-linear on land flow regime could be implemented by a concept of equivalent roughness coefficients (Imamura 2009). 


\section{Model setup and discretization}

Figure 5 shows a sketch of the artificial beach. In the following, typical length scales of prototype magnitudes are considered. The slope of the beach is fixed to 1:20, 1:50 and 1:100 respectively. The water depth at the left domain boundary is fixed to $d=30.00 \mathrm{~m}$. The model is divided into two adjacent sections. The first section is characterized by a uniform water depth whereas the second section resembles the constantly sloping beach profile. The beach is sustained at the shoreline to an elevation of $20.00 \mathrm{~m}$ above sea level to study wave-run up effects. The length of the even bottom section is set to $600.00 \mathrm{~m}$ and the horizontal distance from the base point of the slope to the shoreline amounts to $1 / \alpha \cdot d$ respectively, where $\alpha$ is the beach slope and $d$ is the water depth. At the open boundary time history of sea surface height and horizontal momentum is introduced. Simulations are constantly operated for $t=1000.00 \mathrm{~s}$. Further, we have designed the study domain to be of variable width in order to examine the influence of macro roughness elements (e.g. buildings) and to investigate an identical number of houses normal to the wave propagation direction. Free space between roughness elements (called streets) leading to canalized flow with amplified wave-induced currents is varied likewise. In this study different numbers of macro-roughness element rows are used (1, 5, 10 rows). The proposed simplistic element configurations and element numbers are less realistic than equivalent real urban structure in prototype scale. Yet, for gaining fundamental knowledge of flow and impact characteristics at densely populated coasts such generalizations and simplifications seem admissible and straightforward.

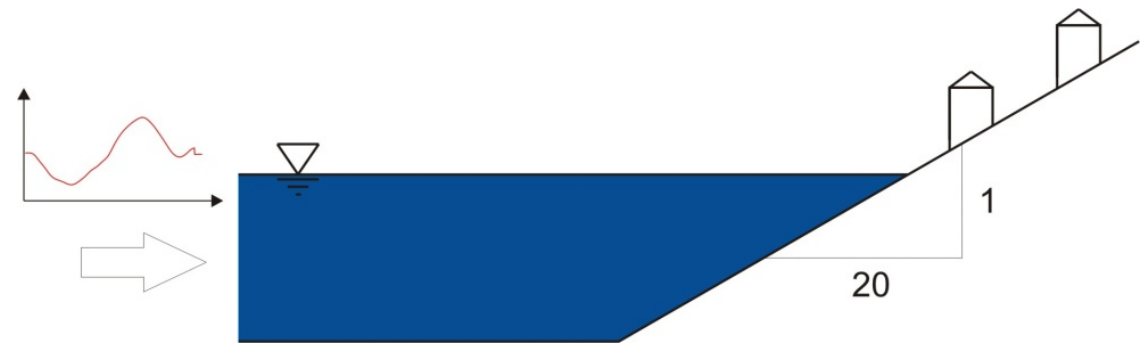

Figure 5. Methodical sketch of the model setup used in the numerical model, beach slope 1:20 and 1:40 respectively, macro-roughness element configurations on the beach according to scenario runs

The hydrodynamic inundation modeling tool used for the detailed run-up simulations is developed and constantly improved by the Australian National University and Geoscience Australia (ANUGA). A finite-volume approach is used to solve the nonlinear shallow water wave equations in conservative form. A spatially varying triangular mesh is used to discretize the area of interest. The conserved quantities are water depth and horizontal momentum in $\mathrm{x}$ and $\mathrm{y}$ direction (Nielsen et al. 2005). The friction term is modeled using Manning's resistance law. A robust algorithm is obtained by employing piecewise linear vector function of $x$ and $y$ for the conserved quantities that allows discontinuities across the edges of a cell while slope limiting functions assure numerical stability. A detailed description of the applied numerical scheme can be found in Zoppou and Roberts (1999).

The choice of the numerical modeling tool has been basically determined by the robustness of the numerical scheme in the wetting and drying area as well as when supercritical flow conditions are expected. Nevertheless, it is admitted that at the same time the absence of a proper eddy viscosity formulation in the equations establishes a deficiency since turbulent effects especially in the wake of the macro-roughness elements are considerably underestimated. Detailed investigations in the context of macro-roughness effects in regard to horizontal and vertical turbulence remain and are being dealt with in present investigations by the authors and other researchers.

\section{Mesh properties}

Mesh generation has been handled by the Triangle tool (Shewchuk 1996). The Triangle tool is implemented in the framework of ANUGA. It uses boundary polygons, inner polygons for refinements and general constraints on triangle areas as well as minimum angles. Areas of domain-wide triangles are limited to $5.00 \mathrm{~m}^{2}$ whereas triangle areas around macro-roughness elements comprise $0.25 \mathrm{~m}^{2}$. The refined region around the elements is defined by an inner and outer polygon relative to the macroroughness element polygon. Outer polygons are at a distance of $0.90 \mathrm{~m}$ and the inner polygons reside $1.00 \mathrm{~m}$ away from the house polygon. In a second step and after the meshing took place, the mesh nodes contained in the macro-roughness polygon are elevated to $20.00 \mathrm{~m}$ elevation above the local ground level in order to resemble the obstacle height in the prototype. Lower elevations, especially 
below the near shore wave amplitude firstly led to an overflow of the obstacles that caused difficulties with the CFL based time stepping in the numerical tool. Obstacle walls appear in the numerical domain as inclined surfaces. Surface angles are approximately $87.0^{\circ}$. This fact needs to be taken into account, when numerical and experimental results are compared since discharge sections between obstacles vary slightly. Figure 6 depicts four of the investigated macro-roughness element configurations for an identical street width. Long wave-induced flow direction is from the left to right. A varying number of 6 to 10 mesh elements are kept in order to adequately model the flow through the macro-roughness elements.
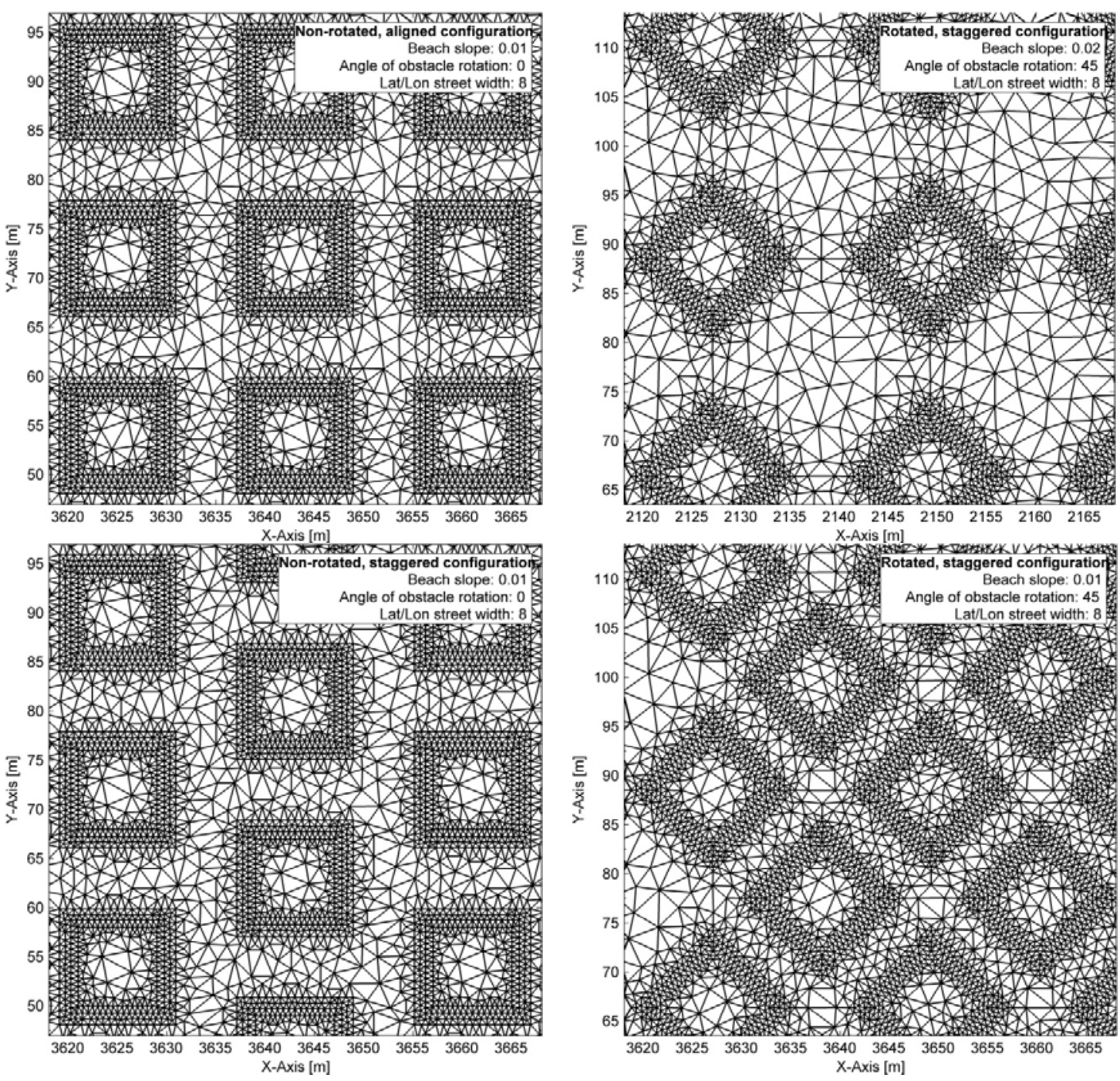

Figure 6. Mesh properties at and around the macro-roughness elements in the computational domain for different combinations: (upper left) Aligned and non-rotated, (upper right) Aligned and rotated, (lower left) Staggered and non-rotated, (lower right) Staggered and rotated, domain is only displayed in parts

Table 1 additionally illustrates the statistical parameters of the hydrodynamic simulation runs. In total, 161 model runs were accomplished and further runs were conducted for sensitivity studies. The number of mesh cells varies from 90434 to 409532 and running times change proportionally to the number of mesh cells. 


\begin{tabular}{|l|l|l|l|l|}
\hline \multicolumn{6}{|l|}{ Table 1. Details of the accomplished simulation runs } \\
\hline Combination & Slope & $\begin{array}{l}\text { Number of } \\
\text { cells }\end{array}$ & Delta t [s] & Runtime [s] \\
\hline Non-rotated, aligned & $1 / 20$ & 90434 & $10^{-2}-10^{-4}$ & 12554.62 \\
& $1 / 50$ & 154432 & $10^{-2}-10^{-4}$ & 28708.04 \\
& $1 / 100$ & 246176 & $10^{-2}-10^{-4}$ & 45316.47 \\
\hline Rotated, aligned & $1 / 20$ & 123068 & $10^{-2}-10^{-4}$ & 29979.70 \\
& $1 / 50$ & 220241 & $10^{-2}-10^{-4}$ & 44167.26 \\
& $1 / 100$ & 409532 & $10^{-2}-10^{-4}$ & 61788.83 \\
\hline Non-rotated, staggered & $1 / 20$ & 95059 & $10^{-2}-10^{-4}$ & 15865.87 \\
& $1 / 50$ & 154449 & $10^{-2}-10^{-4}$ & 32267.37 \\
& $1 / 100$ & 269668 & $10^{-2}-10^{-4}$ & 148995.46 \\
\hline Rotated, staggered & $1 / 20$ & 129162 & $10^{-2}-10^{-4}$ & 25544.93 \\
& $1 / 50$ & 194256 & $10^{-2}-10^{-4}$ & 41011.30 \\
& $1 / 100$ & 331018 & $10^{-2}-10^{-4}$ & 65782.16 \\
\hline
\end{tabular}

Figure 7 illustrates convergence of the normalized run-up depending on the mesh triangle area. In order to find an optimum between computational accuracy and cost, mesh sensitivity was subsequently tested for nine mesh triangle areas. Therefore, an area of $5.00 \mathrm{~m}^{2}$ is determined to yield a good balance between both constraints. Smaller triangles below the given threshold are still present in the vicinity of macro-roughness polygons due to the inner angle constraint.

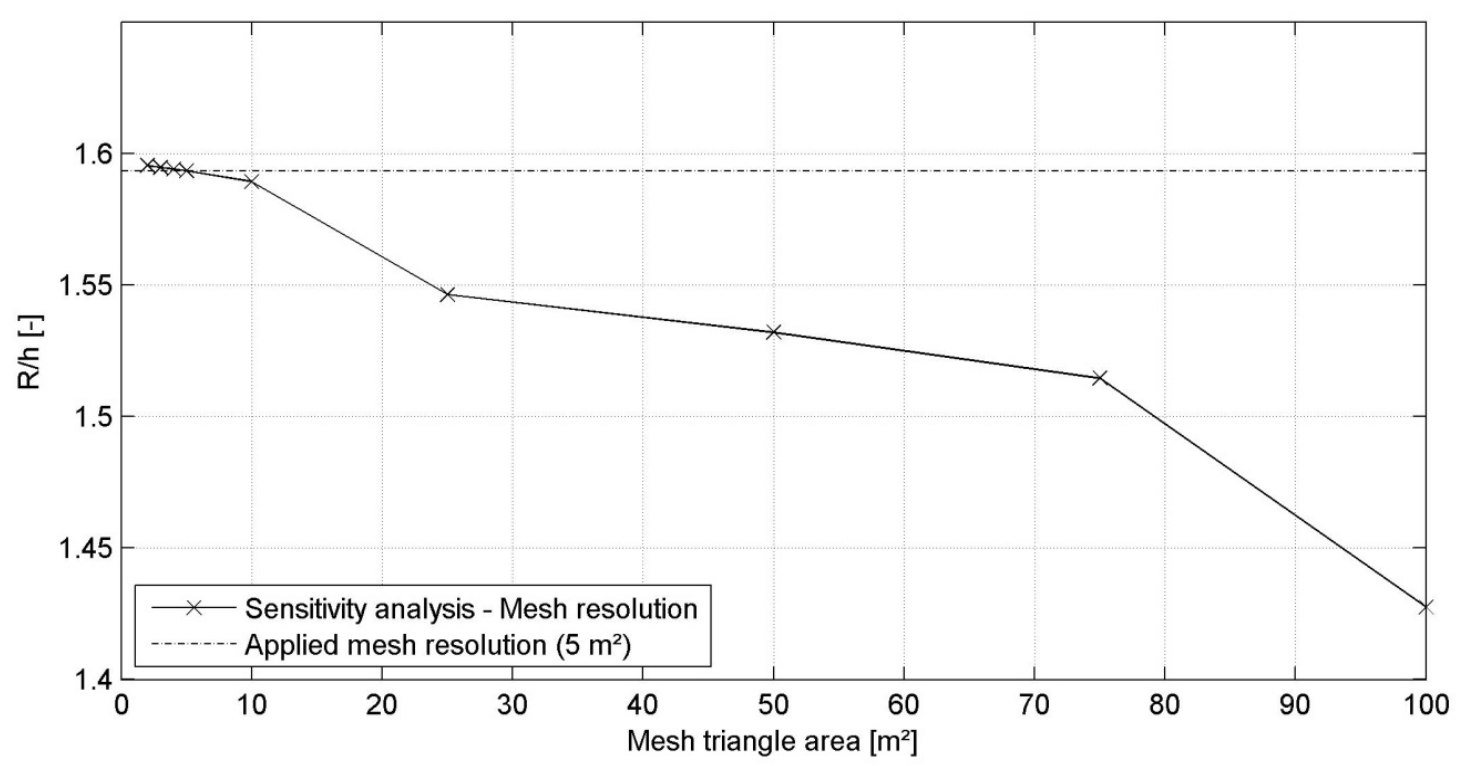

Figure 7. Normalized run-up convergence due to mesh resolution and applied mesh resolution of $5 \mathrm{~m}^{2}$ for the numerical study

\section{Obstruction measure}

In order to allow for a quantitative measure of the effectively obstructed coastal area, an obstruction factor has been defined in Eq. 1 to integrate all relevant structural influences. A linear approach is proposed for the current study even though results indicate that non-linear correlation needs to be considered in further investigations. Since only a first set of experimental studies is completed and validation of the numerical model is still under progress, linear dependency for the grouping factor is used instead. Eq. 1 gives the functional relation:

$$
\alpha_{\text {obstr }}=f\left(A_{\text {eff_cs }}^{*}, n_{\text {ob_rows }}, \beta_{\text {group }}\right)
$$

$A_{\text {eff_cs }}^{*}$ reflects the effective cross sectional area, $n_{o b_{-} \text {rows }}$ the number of obstruction rows parallel to the shore line and $\beta_{\text {group }}$ depicts a grouping factor according to table 2 . The formula for the calculation of the obstruction factor reads 


$$
\alpha_{\text {obstr }}=n_{h_{-} \text {lat }} \cdot \frac{b_{\text {house }}}{b_{\text {flume }}} \cdot n_{\text {ob_rows }} \cdot \beta_{\text {group }}
$$

Furthermore the effective cross sectional area $A_{\text {eff_cs }}^{*}$ is investigated, where $n_{h_{-} \text {lat }}$ is the numbers of houses lateral to the wave propagation direction, $b_{\text {house }}$ is the width of an individual house and $b_{\text {flume }}$ is the width of either the numerical or the experimental flume. The definition of the obstruction measure assures that at higher values of the obstruction factor the run-up is reduced, whereas lower values allow for greater permeability with respect to the flow. Different macro-roughness element configurations are subsequently connected to grouping factor distribution shown in the table assuming a linear influence as a start.

\begin{tabular}{|c|c|c|}
\hline Reference & Combination & Grouping factor \\
\hline$(1)$ & Non-rotated, aligned & 0.025 \\
\hline (2) & Non-rotated, staggered & 0.075 \\
\hline (3) & Rotated, aligned & 0.05 \\
\hline (4) & Rotated, staggered & 0.10 \\
\hline
\end{tabular}

The following section presents numerical results contrasting first quantitative and qualitative experimental results, which will be calibrated against the presented laboratory experiments in the future.

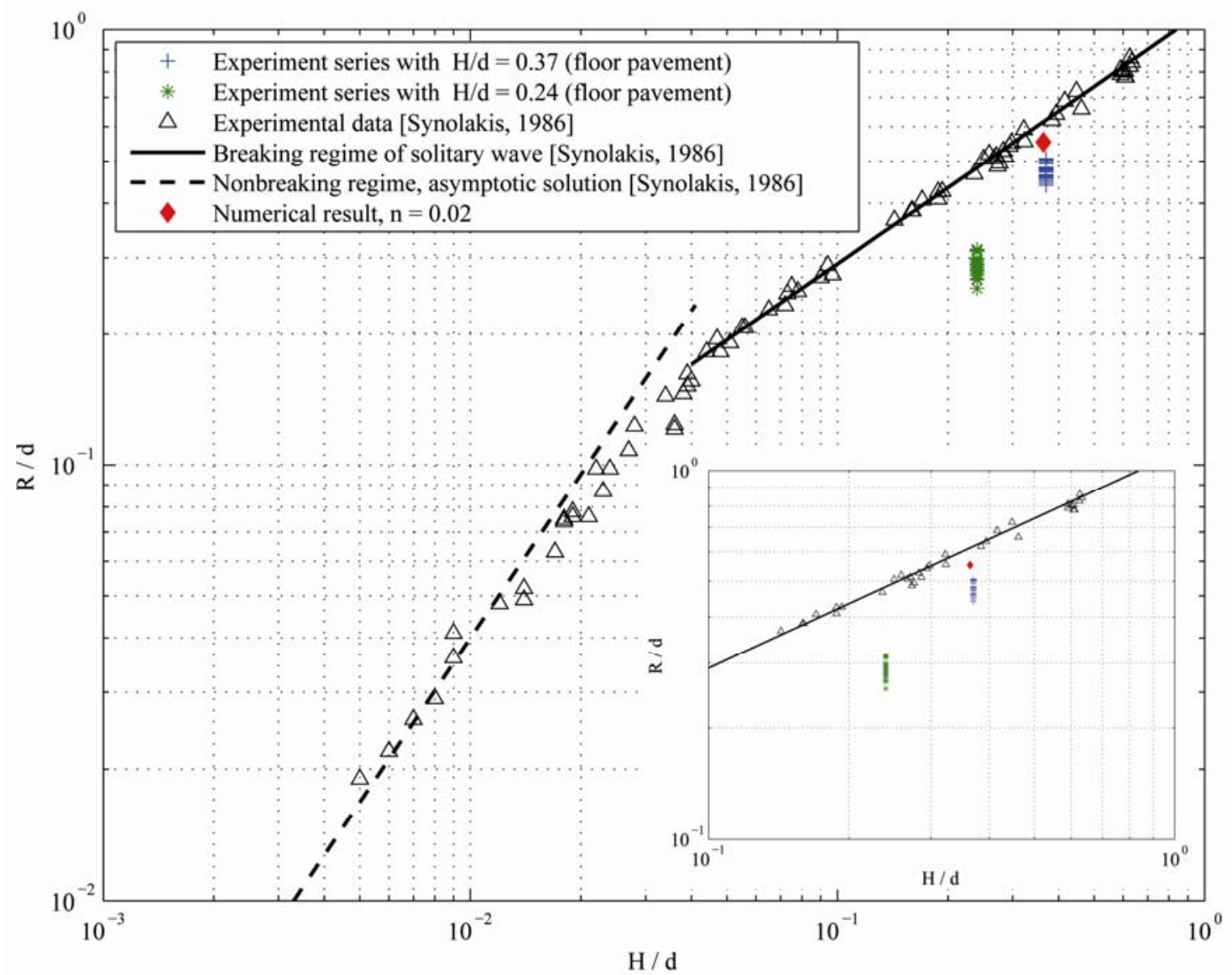

Figure 8. Comparison of experimental results with solitary waves (Synolakis 1986) with experiments applying wave shapes e.g. shown in figure 3 under laboratory conditions discussed in the physical modeling section of this paper, finally numerically deduced wave run-up (red diamond) for a Manning's $n$ value of 0.02 , insert highlights the important value range $10^{-1}<R / d<10^{0}, 10^{-1}<H / d<10^{0}$ 


\section{RESULTS AND DISCUSSION}

Resulting run-up values from either numerical or experimental studies need to be put into context with former investigations of long wave run-up. A widely accepted reference was carried out by (Synolakis 1986). Figure 8 displays experimental and numerical results according to the previous sections related to the reference study. To date, two relative wave heights have been investigated. Relative runup of waves under consideration is clearly below solitary wave run-up. What is more, no wave breaking took place even for the highest wave heights.

Wave run-up heights deduced from numerical modeling based on NSWE with a bottom roughness value of $n=0.02$ slightly overestimate results from the experimental study, where a flat floor pavement was used for the 1:20 sloping beach wedge. Further, re-iterations of the numerical simulations with higher Manning values could reduce the difference between experimental and numerical results.

Run-up dynamics of the approaching long wave interaction with macro-roughness is qualitatively characterized in figure 9. On the left, the wave front approaches the first obstacle row, where partial wave reflection takes place. Reflections are dependent on the blocking rate of the discharge section at the first obstacle row. Further increase in water levels leads to flow constrictions and an accelerated flow in between the building objects resembling the typical Venturi-effect known from fluid mechanics. The flow changes from sub- to supercritical flow at this stage while the reflected wave is traveling seaward. The highlighted pattern of flow dynamics is successively repeated at every next obstacle row, but with reduced water levels and less prominent amplified currents in between the obstacles. Flow separation exists at vertical obstacle edges whereas it is less pronounced when obstacles are $45^{\circ}$ rotated.
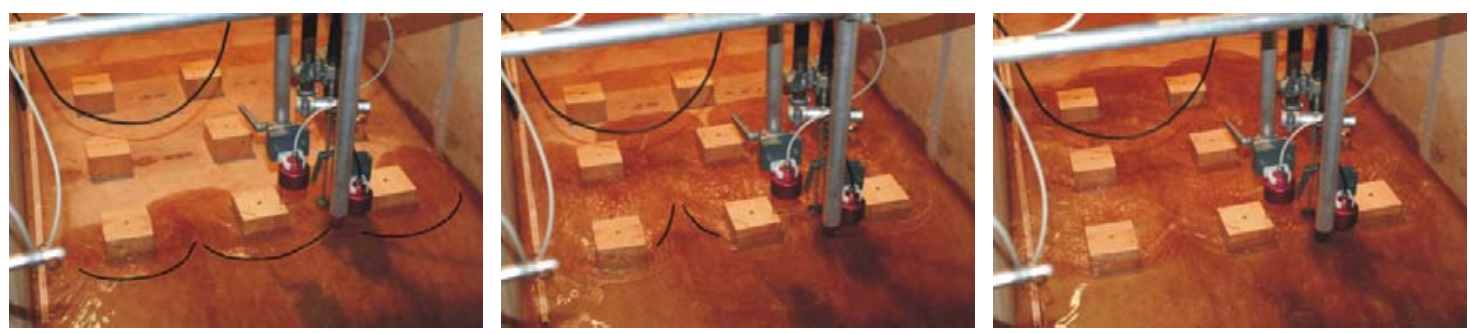

Figure 9. Three snapshots of the run-up process in chronological order from left to right, obstacles consist of wooden blocks with edge length of $10 \mathrm{~cm}$ at a distance of $15 \mathrm{~cm}$

Figure 10 at last illustrates the correlation of normalized wave run-up in regard to the obstruction factor as outlined above for the four analyzed configurations of macro-roughness elements. Colorbars indicate the spacing in between the obstacles. First interpretation leads to the outcome that normalized run-up heights are diminished if either spacing between the obstacles is reduced or number of obstacle rows is increased. In addition, figure 10 (a) displays the run-up magnitudes computed for the mesh size sensitivity study. Highest reduction is usually observed where obstacles are distributed in a staggered manner (comp. fig. 6). Nevertheless, an exception is given for the rotated, aligned configuration shown in figure 10 (c). The result pattern is similar for one row of obstacles in regard to the remaining three configurations but for 5 and 10 obstacle rows a higher wave run-up is found instead. Further tests and repetitions are needed to clarify interpretation for these surprising results. Further, additional simulation runs are required using different beach slopes, rows of obstacles as well as street widths between the obstacles in order to expand the data basis and to deduce a reasonable correlation for the analyzed quantities. Further studies are currently in progress.

\section{CONCLUSION AND SUMMARY}

An alternative methodology in long wave generation has been successfully tested. Arbitrary long waves can be generated by means of a PID controller combined with a set of high-capacity pipe pumps. The experimental procedure has been applied to long wave run-up at a 1:20 sloping beach. Subsequently, the results serve as calibration data set for numerical modeling of the coastal situation. A comparison to solitary wave run-up yields significantly lower values as well as differences in the breaking characteristics (no breaking). The wave generation methodology suits similarly well when long wave run-up interacting with solid obstacles on the beach should be examined. 

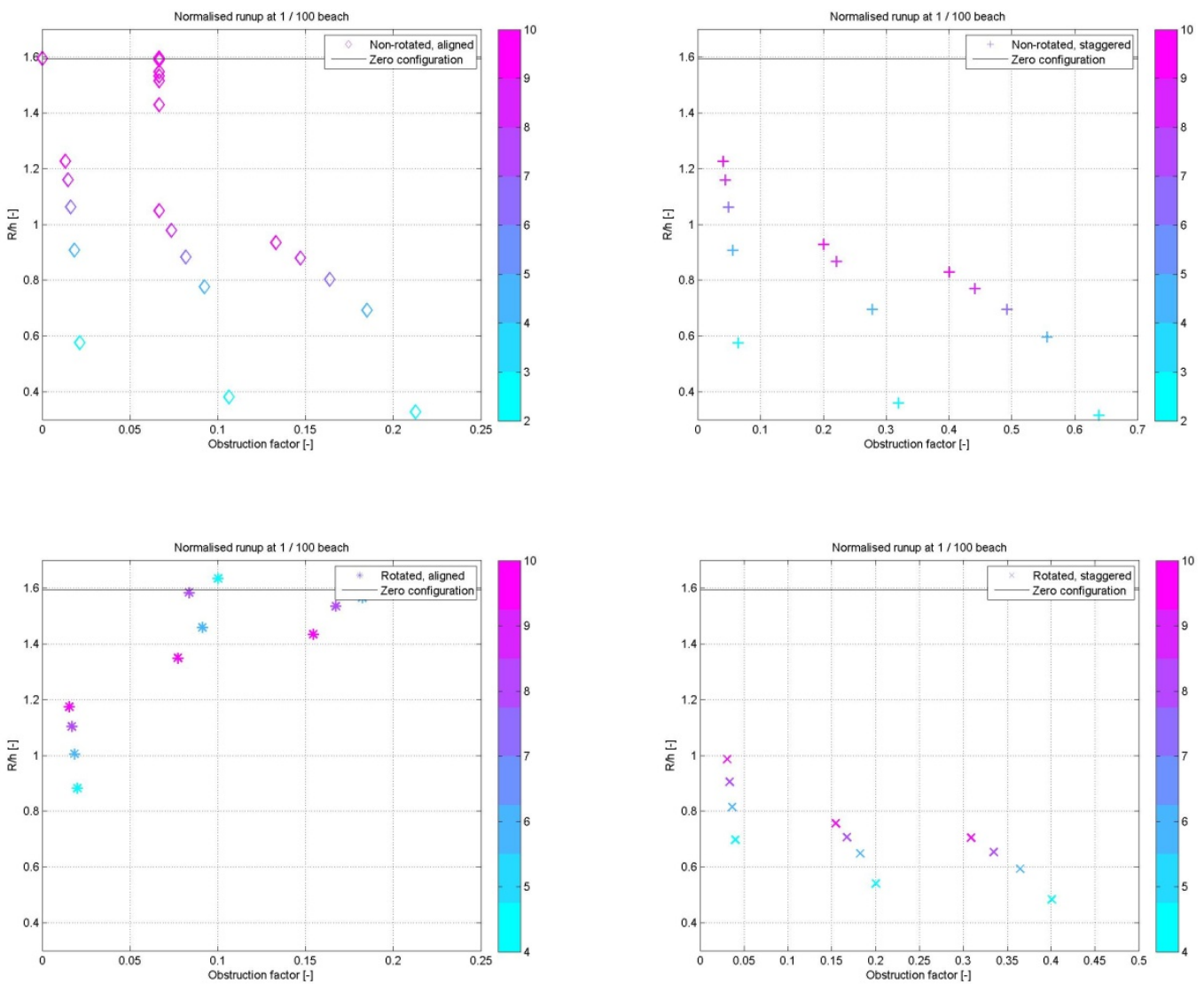

Figure 10. Representative run-up distributions due to different macro-roughness combinations against obstruction factor at a slope of $1 / 100$, color bar color codes the spacing between macro-roughness elements, (a) upper left: non-rotated and aligned, (b) upper right: non-rotated and staggered, (c) lower left: rotated and aligned and (d) lower right: rotated and staggered

First results from numerical modeling also indicate that a correlation exists between wave run-up and the presence of macro-roughness elements on the beach resulting in a certain obstruction of the beach cross section even though only linear correlation has been considered so far. Also, validation and calibration against physical model test remains for further analysis and to test if correlation relations hold true.

Since the applied numerical model lacks a suitable eddy viscosity formulation, the influence of the energy dissipation during wave run-up is neglected. This matter needs to be addressed in follow-up studies. Furthermore, numerical modeling usually does not account for volume losses which are due to storage of water inside obstacles (houses, buildings, etc.). The order of magnitude of this effect has to be assessed in future research and feasible numerical routines have to be developed as well.

\section{ACKNOWLEDGMENTS}

The authors would like to thank various colleagues from the costal engineering community for their valuable comments and questions leading to ongoing fruitful discussion during and after the conference presentation.

\section{REFERENCES}

Åström, K. J., and R. M. Murray. 2008. Feedback Systems - An Introduction for Scientists and Engineers. Princeton University Press.

Briggs, M.J., C.E. Synolakis, G.S. Harkins, and D.R. Green. 1995. Laboratory experiments of tsunami runup on a circular island. Pure and applied geophysics 144, no. 3-4: 569-593.

Cox, Daniel, Takashi Tomita, Patrick Lynett, and Rob Holman. 2009. Tsunami inundation with macroroughness in the constructed environment. In Proceedings of the International Conference on 
Coastal engineering, 2:1421-1432. World Scientific.

Galvin, C. J. 1964. Wave-height prediction for wave generators in shallow waters. March.

Goseberg, N., and T. Schlurmann. 2008. Relevant factors on the extent of inundation based on tsunami scenarios for the city of Padang, West Sumatra. In Proceedings of the International Conference on Tsunami Warning (ICTW).

Goseberg, N., and T. Schlurmann. 2009. Enhanced hazard mapping on a medium-resolved numerical grid for the city of Padang, West Sumatra. Journal of ship technology 5, no. 2 (July): 13-21.

Goseberg, N., A. Stahlmann, S. Schimmels, and T. Schlurmann. 2009. Highly-resolved numerical modeling of tsunami run-up and inundation scenarios in the city of Padang, West Sumatra. In Poster Proceedings of the International Conference on Coastal engineering, 27-40.

Hashimoto, H., and K. Park. 2008. Flood Recovery, Innovation and Response I. WIT press.

Imamura, F. 2009. Tsunamis: Ideas and Observations on Progress in the Study of the Seas. Harvard Univ. Press.

Lynett, Patrick J., Tso-Ren Wu, and Philip L.-F. Liu. 2002. Modeling wave runup with depthintegrated equations. Coastal Engineering 46 (July): 89-107(19).

Madsen, P.A., D.R. Fuhrman, and H.A. Schäffer. 2008. On the solitary wave paradigm for tsunamis. Journal of Geophysical Research C: Oceans 113, no. 12: 1-22.

Nielsen, O., S. Roberts, D. Gray, A. McPherson, and A. Hitchman. 2005. Hydrodynamic modelling of coastal inundation. In MODSIM 2005 International Congress on Modelling and Simulation, 518523.

Pedro, H.T.C., K.-W. Leung, M.H. Kobayashi, and H.R. Riggs. 2007. Numerical study of the wave impact on a square column using large Eddy simulation. In Proceedings of the International Conference on Offshore Mechanics and Arctic Engineering - OMAE, 3:1015-1021.

Shewchuk, Jonathan Richard. 1996. Triangle: Engineering a 2D Quality Mesh Generator and Delaunay Triangulator. In Applied Computational Geometry: Towards Geometric Engineering, 1148:203222. Springer-Verlag, May.

Soares-Frazao, S., J. Lhomme, V. Guinot, and Y. Zech. 2008. Two-dimensional shallow-water model with porosity for urban flood modelling. Journal of Hydraulic Research 46, no. 1: 45-64.

Soares-Frazao, S., and Y. Zech. 2008. Dam-break flow through an idealised city. Journal of Hydraulic Research 46, no. 5: 648-658.

Synolakis, C. E. 1986. The runup of long waves. W. M. Keck Laboratory of Hydraulics and Water Resources, California Institute of Technology, December.

Taubenböck, H., N. Goseberg, N. Setiadi, G. Lämmel, F. Moder, M. Oczipka, H. Klüpfel, et al. 2009. Last-Mile preparation to a potential disaster - Interdisciplinary approach towards tsunami early warning and an evacuation information system for the coastal city of Padang, Indonesia. Natural Hazards and Earth System Sciences (NHESS) 9, no. 4: 1509-1528.

Tomita, Takashi, Kazuhiki Honda, and Taro Kakinuma. 2007. Application of three-dimensional tsunami simulator to estimation of tsunami behaviour around structures. In Proceedings of the International Conference on Coastal engineering, 2:1677-1688. World Scientific.

Ward, S. N., and S. Day. 2008. Tsunami balls: A granular approach to tsunami runup and inundation. Communications in Computational Physics 3, no. 1: 222-249.

Wijetunge, J. J. 2010. Numerical Simulation of the 2004 Indian Ocean Tsunami: Case Study of Effect of Sand Dunes on the Spatial Distribution of Inundation in Hambantota, Sri Lanka. Journal of Applied Fluid Mechanics 3, no. 2: 125-135.

Xiao, H., and W. Huang. 2008. Numerical modeling of wave runup and forces on an idealized beachfront house. Ocean Engineering 35, no. 1: 106-116.

Zoppou, C., and S. Roberts. 1999. Catastrophic Collapse of Water Supply Reservoirs in Urban Areas. Journal of Hydraulic Engineering 125, no. 7: 686-695. 